\title{
Space Charge Properties of UHMWPE/OibPOSS Composites
}

\author{
Zhiqiang $\mathrm{Xu}$ and George Chen \\ Department of Electronics and Computer Science \\ University of Southampton \\ Southampton, UK \\ zhq.xu@soton.ac.uk
}

\author{
Meng Guo and Éric David \\ Department of Mechanical Engineering \\ École de technologie supérieure \\ Montréal, Québec, Canada
}

\author{
Michel Fréchette \\ Department of Material Science \\ Institut de recherche d'Hydro-Québec \\ Varennes, Québec, Canada
}

\begin{abstract}
POSS (Polyhedral Oligomeric SilSesquioxanes) is a type of filler of great interest in the field of polymeric micro and nanodielctrics. The thermal and mechanical properties after incorporation of POSS into polymer via chemical cross-linking or physical blending have been paid much attention to recently. In this paper, the space charge property of microstructured composites consisting of UHMWPE (ultra-high-molecular weight polyethylene) and OibPOSS (OctalsoButyl Polyhedral Oligomeric SilSesquioxanes) was studied. The high-energy shaker mill was used to obtain the composites. Briefly this paper show the space charge profiles for samples with $0 \mathrm{wt} \%$, $1 w t \%, 5 w t \%$ OibPOSS contents. The charge injection in all the samples under different applied voltages is not significant. Some homocharge can be observed from the interface between electrode and sample, only in the case of $1 \mathrm{wt} \%$ and $5 \mathrm{wt} \%$ composites. The charge injection and decay processing is inactive in the composites samples.
\end{abstract}

Keywords- UHMWPE, ultra-high-molecular weight polyethylene, Octaisobutyl polyhedral oligomeric silsesquioxanes, POSS, OibPOSS, ball milling, PEA, Space charge,

\section{INTRODUCTION}

Nanodielectrics is a popular term in dielectric community and lots of attention were paid to this subject. POSS (Polyhedral Oligomeric SilSesquioxanes) is not a typical nano filler such as clay, alumina and silica, but a filler of great interest in the field of polymeric micro and nanodielctrics [1,2]. Despite of the fact that they are by nature molecules of nanoscaled sizes, a majority of them exist as crystalline solids. One of the challenges of this material's investigation and application is the dispersing of POSS within polymer matrix at nanoscale.

Typically, POSS are cage-structured silicon-oxygen backbone surrounded by one or more types of functionalities. A reactive functionality favors chemical bonding between POSS and the polymer matrix, whereas an unreactive functionality merely renders physical mixing between them[3,4]. Unreactive OctaIsoButyl POSS (OibPOSS) (Fig. 1) is studied in this paper.

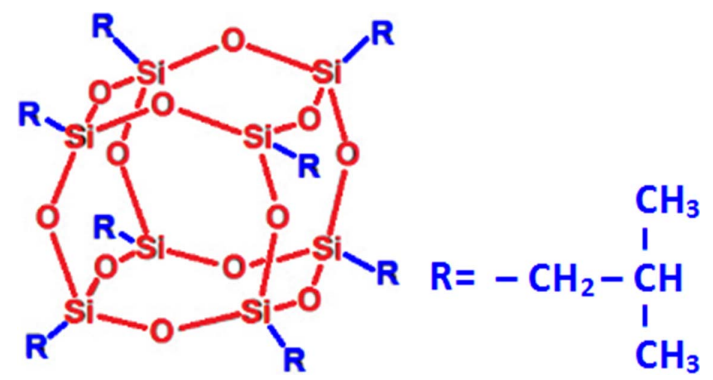

Fig. 1 chemical structure of POSS with isobutyl group [5]

Compared to the studies of thermal and mechanical properties of PE-based nanocomposites containing unreactive POSS, the dielectric property still needs a lot of attention. The space charge property of microstructured composites consisting of ultra-high-molecular weight polyethylene (UHMWPE) and OibPOSS was studied in this paper.

The high-energy shaker mill was used to obtain the composites. Three kinds of UHMWPE/OibPOSS composites were made with $100 / 0 \%, 99 / 1 \%$ and $95 / 5 \%$ mass fraction. Plaque samples with $\sim 0.23 \mathrm{~mm}$ thickness were compression moulded afterwards. The space charge distribution was measured using the pulsed electroacoustic technique (PEA) which is widely used in the solid dielectrics study around the world. The applied voltage on the sample were selected from $10 \mathrm{kV} / \mathrm{mm}$ to $40 \mathrm{kV} / \mathrm{mm}$. 


\section{EXPERIMENT SETUP}

\section{A. Sample fabrication}

Ball milling was attended in order to produce nano dielectrics having nanoscaled OibPOSS embedded within the PE matrix. With several zirconia balls serving as the grinding medium, UHMWPE powder and OibPOSS powder (mass fractions: $100 / 0 \%, 99 / 1 \%$ and $95 / 5 \%$ ) were ball milled in a zirconia vial for $30 \mathrm{~min}$. The processing of ball milling was described in detail in [5].

The three types of composite powders obtained from ball milling were subsequently subjected to compression moulding. The material of interest was kept in 15 ton and $150^{\circ} \mathrm{C}$ for 15 minutes in the specific-sized mould, and consequently, three plate samples of $\sim 0.23 \mathrm{~mm}$ were obtained.

The resulting UHMWPE plate, UHMWPE/OibPOSS (99/1) plate, and UHMWPE/OibPOSS (95/5) plate were subjected to space charge measurements.

The morphology of these samples were studied by scanning electron microscopy. Despite of the attempt to use ball milling as a method for producing OibPOSS-containing nanodielectrics, OibPOSS was found to disperse at micron scale in both the $1 \mathrm{wt} \%$ and the $5 \mathrm{wt} \%$ composites. More detailed morphological characteristics of the composites can be found in [5].

\section{B. Experiment equipment}

The pulsed electroacoustic (PEA) technique was used to investigate the space charge profile. PEA has been considered as the most effective technique for measuring space charge in solid dielectrics. The measuring system for the present research has a good spatial resolution and sensitivity because a thin PVDF piezoelectric sensor and a narrow width pulse generator was used.

Only positive voltage was applied to the samples in this study, the lower cathode is aluminium electrode and the upper anode is semiconducting polymer electrode. All the experiments were carried out at room temperature and under controlled humidity. Space charge distribution was taken at various times during volts-on conditions with electric field range from $10 \mathrm{kV} / \mathrm{mm}$ to $50 \mathrm{kV} / \mathrm{mm}$ and the decay results after the removal of the electric field were also monitored.

\section{EXPERIMENTAL RESULTS AND DISCUSSION}

The applied voltage added to the sample (around $0.2 \mathrm{~mm}$ thickness) increased from $2 \mathrm{kV}$ to $10 \mathrm{kV}$ and the tests were carried out for 10 minutes in 2 minute steps.

\section{A. UHMWPE100\%+0\%OibPOSS}

Fig. 2 shows the space charge density distribution of UHMWPE sample under applied voltage of $2 \mathrm{kV}$ to $10 \mathrm{kV}$. The negative peak and positive peak, which means the charge captured at cathode and anode, increased with the applied voltage. Only small but can't be ignored hetero-charge package appeared in the area of next to the anode.

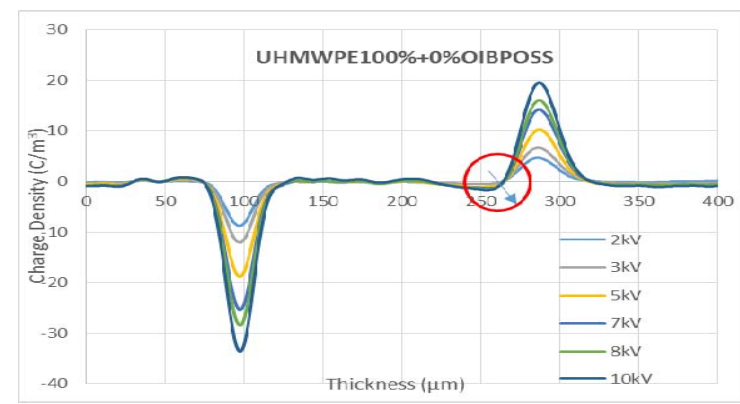

Fig. 2 the space charge distribution in UHMWPE sample.
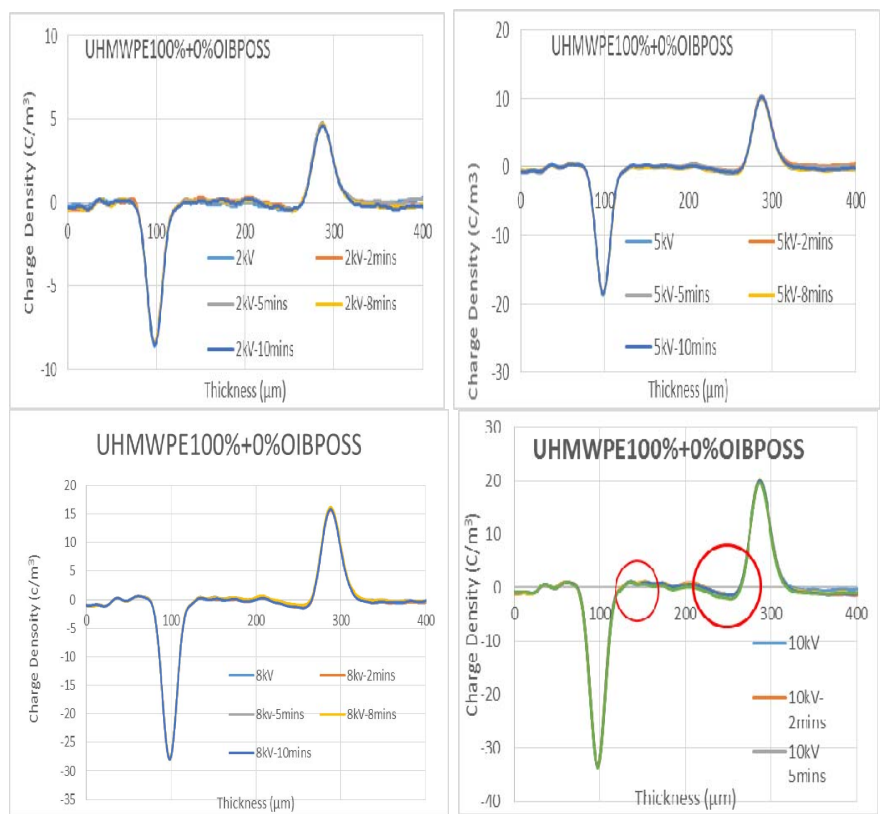

Fig. 3 space charge distribution of UHMWPE sample under $2 \mathrm{kV}, 5 \mathrm{kV}, 8 \mathrm{kV}$, $10 \mathrm{kV}$ applied voltage.

The four pictures shown in Fig. 3 are the charge density profiles of UHMWPE under different applied voltage of $2 \mathrm{kV}$, $5 \mathrm{kV}, 8 \mathrm{kV}$ and $10 \mathrm{kV}$. It is obvious that the injected charges in the material are very small in all level of stress and no change with the duration of voltage application. The small package of the hetero-charge near anode was found and the amount of them enhanced a tiny bit when applied voltage increase to $10 \mathrm{kV}$.

$10 \mathrm{kV}$ applied voltage was removed after applying 20 minutes, and the charge distribution across the sample were recorded and shown in Fig. 4. There are some hetero-charge appearing in the sample next to both electrodes. Normally the charge will keep dropping down till all escaped away when remove the electric voltage, no matter its fast or slow. But only the charge density on the electrodes shows the decay trace in first 30 seconds, $70 \%$ off, and kept stable from 30 second to 15 minutes. However captured hetero-charge in the bulk didn't drop down with time. All these charges are not easy to escape from the trapping site. 


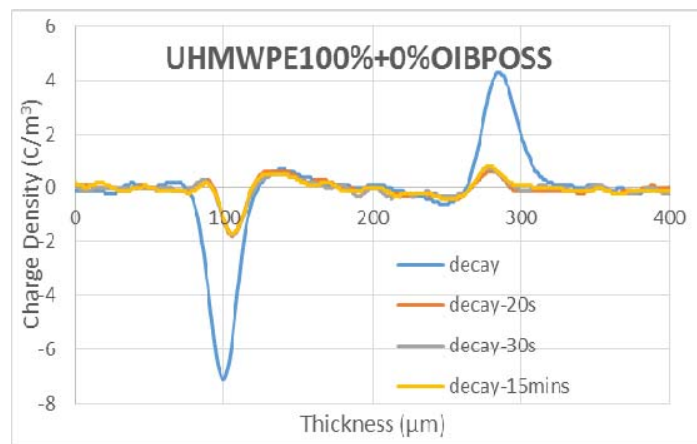

Fig. 4 Decay space charge distribution of UHMWPE sample under $10 \mathrm{kV}$ 20 mins applying.

The charge distribution in UHMWPE is very different from typical LDPE or XLPE [6]. It didn't show remarkable charge accumulated under $10 \mathrm{kV} / \mathrm{mm}$ to $50 \mathrm{kV} / \mathrm{mm}$. and the charge profile change fairly small during 20 minutes stressing time. The charge is not easy to transport through the material.

When the sample was made by ball milling, zirconia grinding balls were used. That is the possible reason contributing to the tiny hetero charge on the surface of the sample when measure the space charge using PEA.

\section{B. UHMWPE99\%+1\%OibPOSS}

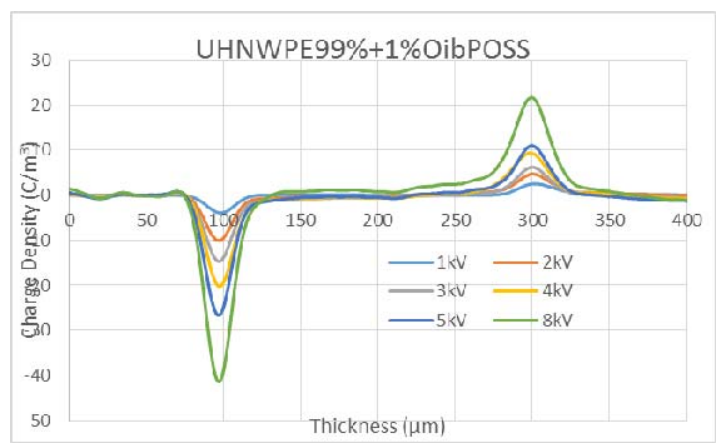

Fig. 5 the space charge distribution in UHMWPE/OibPOSS (99/1) sample.

Fig.5 illustrates the charge distribution in the sample UHMWPE/OibPOSS (99/1) under applied voltage, from $2 \mathrm{kV}$ to $8 \mathrm{kV}$. Homocharge injected from both electrodes are noticeable.

When the applied voltage is under $5 \mathrm{kV}$, both negative charge and positive charge can be found in the bulk of sample, although the amount is small. When the $8 \mathrm{kV}$ applied voltage was added to the sample, the positive charge dominated the bulk area. The movement of charge carriers in the sample is not significant. From the results shown in Fig. 6, charge injection occurred in the beginning of electric field applying, and the net charge density kept stable. For example, charge distriubution is nearly same between results of 2 mins and 3hours under the $8 \mathrm{kV}$ applied voltage. The injection was stucked in one of the situation.

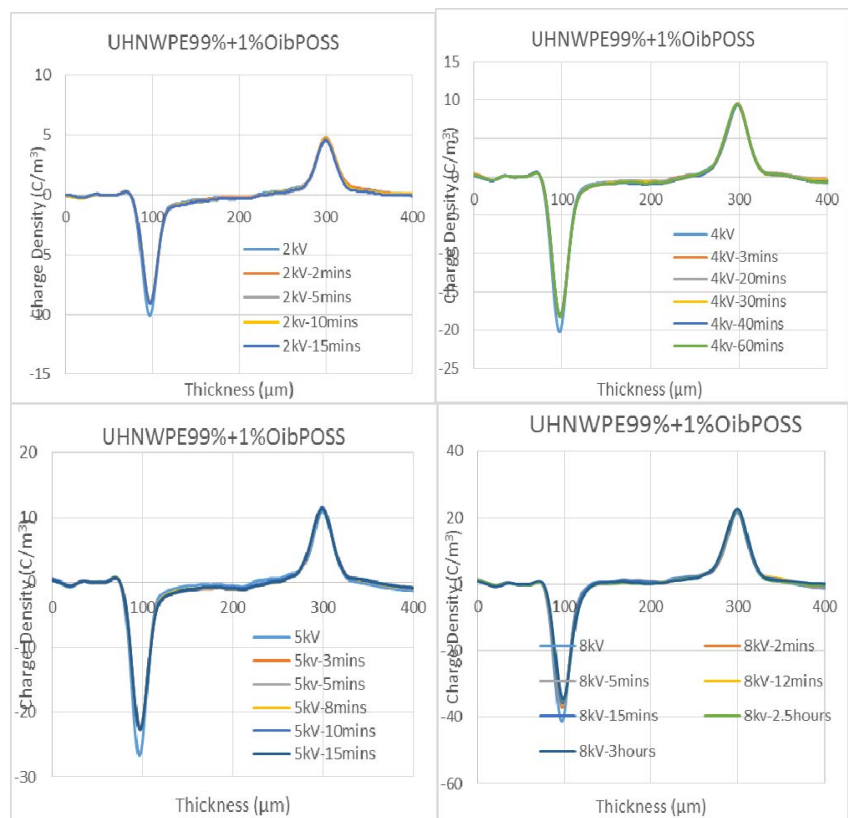

Fig. 6 Space charge distribution in UHMWPE/OibPOSS (99/1) sample under $2 \mathrm{kV}, 4 \mathrm{kV}, 5 \mathrm{kV}$ and $8 \mathrm{kV}$.

Fig. 7 shows the charge decay processing measured after the removal of the applied voltage $8 \mathrm{kV}$ for 3 hours for UHMWPE/OibPOSS (99/1) sample. The results display that the mainly charge left in the bulk is negative charge and there are induced charge captured from electrodes. The peaks at both electrodes decrease regularly with time, $30 \%$ left after 60 mins decay, but the negative charge in the bulk of close to cathode decay very slowly and unremarkably.

The charge injection can be found easier within the sample of UHMWPE/OibPOSS (99/1) than in UHMWPE. Once the charge formed or trapped, it is not easy to change with or without the applied voltage. The OibPOSS filler may change the depth of trapping site in the PE and change the PE chain mobility. And the cage structure of OibPOSS may inhibit the charge injection of space charge.

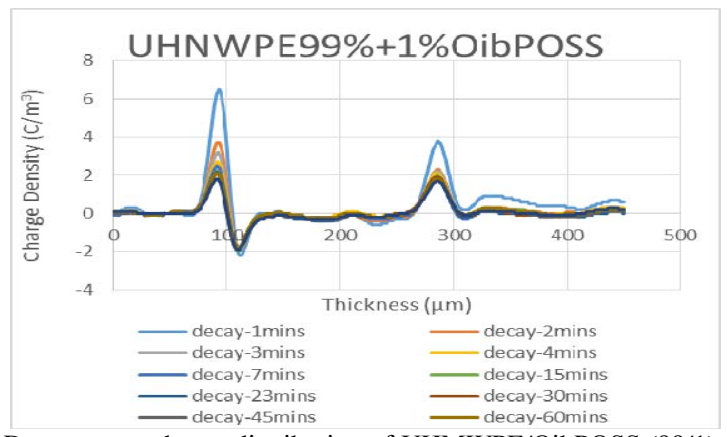

Fig. 7 Decay space charge distribution of UHMWPE/OibPOSS (99/1) sample under $8 \mathrm{kV} 3$ hours applying.

\section{UHMWPE99\%+5\%OibPOSS}

The space charge distribution in the sample of UHMWPE/ OibPOSS (95/5) was study in this section. Fig. 8 gives the charge profile when the applied voltage increased from $2 \mathrm{kV}$ to $10 \mathrm{kV}$. Homo-charge close to anode can be observed very clearly, presented in red circle. 


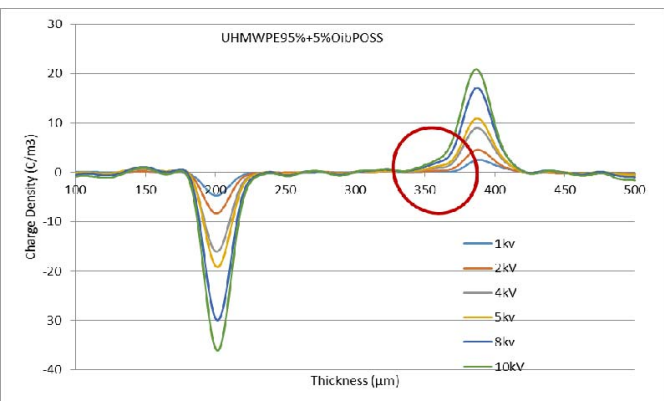

Fig. 8 Space charge distribution of UHMWPE/OibPOSS (95/5) sample.
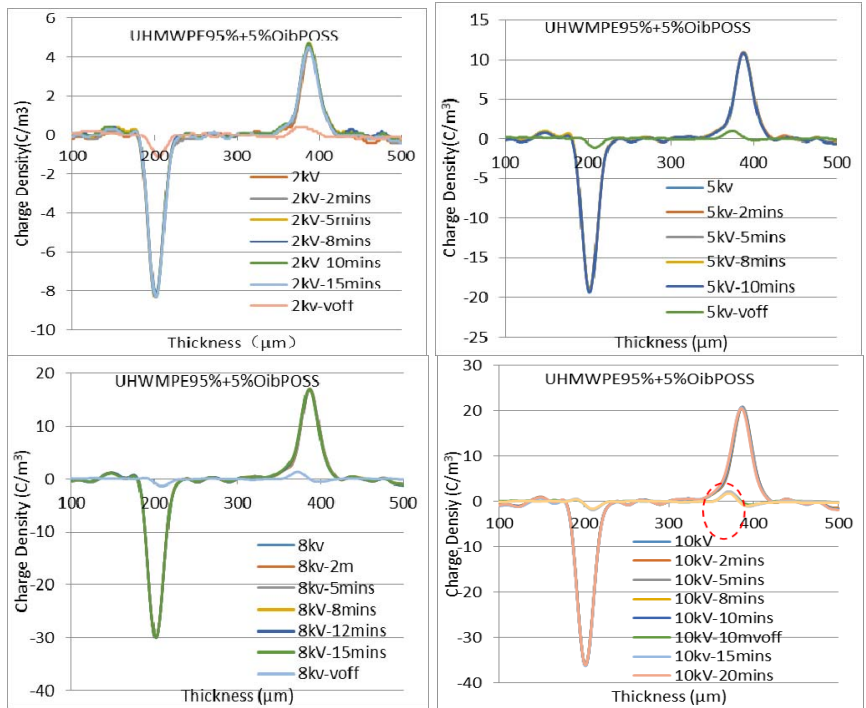

Fig. 9 Space charge distribution in UHMWPE/OibPOSS (95/5) sample under $2 \mathrm{kV}, 5 \mathrm{kV}, 8 \mathrm{kV}$ and $10 \mathrm{kV}$

Fig. 9 shows the volts-on results under different electric field during 15 minutes and volts-off results when applied field removed after 15 minutes. Both Volt-on and Volts-off results can give the information that a small amount of charge accumulated in the sample close to anode. The injected charge can be easily observed and the amount is kept stable during the measuring time.

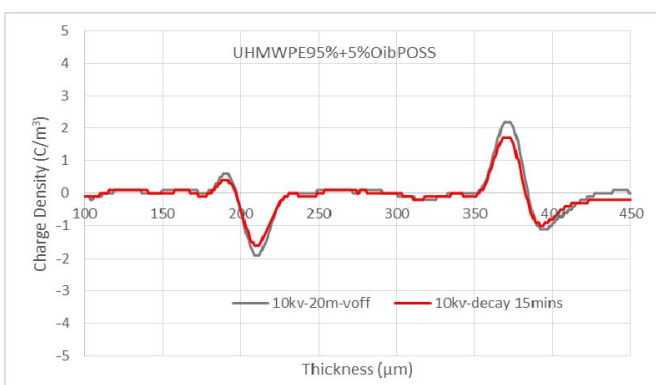

Fig. 10 Decay space charge distribution of UHMWPE/OibPOSS (95/5) after $10 \mathrm{kV} 20 \mathrm{mins}$ applying.

Fig. 10 shows the volt-off and decay results after applying $10 \mathrm{kV} 20 \mathrm{mins}$. There are both positive and negative charge left in the sample in the area of close to anode and cathode which means definitely homo-charge injected in this sample. The injected charge trapped in the material and the trapping level is stable, although the amount is not huge but the small amount charge is very difficult to escape away. Charge density decrease only $20 \%$ in 15 minutes which is shown as the red line in Fig. 10.

The space charge distributions in the UHMWPE/OibPOSS sample are very similar to UHMWPE sample. Charge mobility is not very high, the injected charge is not easy to go further across the sample and hard to decay as well.The charge profile in UHMWPE/OibPOSS shows the difficult injection and difficult ejection. This indictates the major traps in the sample are deep trap and charge carriers are essentially trapped in deep traps.

Because of the crystalline structure of POSS and the micro filler of OibPOSS may change the PE chain mobility and the critical interfacial area may increase the depth of the charge trapping site. Micro filler can act as scattering sites for electrons and introduce the voids at the filler matrix interface. These are the possible reasons of samples with OibPOSS having more charges accumulated than neat UHMWPE sample.

\section{CONCLUSIONS}

Ultra high molecular weight polyethylene (UHMWPE) with and without micro sized OibPOSS samples have been characterised in space charge accumulation in this study. Ball milling is used to produce dielectric composites, containing $0 \mathrm{wt} \%, 1 \mathrm{wt} \%$ and $5 \mathrm{wt} \%$ OibPOSS. Only a small amount of charges can be found from the experiment results in each type of sample. For UHMWPE, only tiny hetero-charge appeard in the area close to the anode. This charge can be brought by the zirconia used when making the samples. For UHMWPE/OibPOSS (99/1), homo charge was found both from the Volts-on and the Volts-off results. And the charges are trapped in deep trap and difficult to escape. For UHMWPE/OibPOSS (95/5), homo charges which can be captured are only around the anode interface area. The more micro filler added, the less charge movement inside the high molecular weight PE sample.

\section{REFERENCES}

[1] D. B. Cordes, P. D. Lickiss, and F. Rataboul, "Recent Developments in the Chemistry of Cubic Polyhedral Oligosilsesquioxanes," Chemical Reviews, vol. 110, pp. 2081-2173, 2010.

[2] M. Joshi and B. S. Butola, "Polymeric NanocompositesPolyhedralOligomeric Silsesquioxanes (POSS) as Hybrid Nanofiller," Journal of Macromolecular Science, Part C, vol. 44, pp. 389-410, 2004.

[3] E. Markovic, K. Constantopolous, and J. G. Matisons, "Polyhedral Oligomeric Silsesquioxanes: From Early and Strategic Development through to Materials Application," in Applications of Polyhedral Oligomeric Silsesquioxanes, ed: Springer,, pp. 1-46. 2011

[4] C. Dearmitt, Pulyhedral Oligometric silsesquioxane Handbook, 2013.

[5] M. Guo, M. Fréchette, E. David, N.R. Demarquette, "PolyethyleneBased Dielectric Composites containing Polyhedral Oligomeric Silsesquioxanes Obtained by Ball Milling", Transiction on Electrical and Electroic Materials Vol 16, pp.53-61, 2015.

[6] M.E. Sepulveda, J.M. Martinez-Tarifa and J.Sanz-Feito, "Ultra High Molecular Weight Polyethylene (UHMWPE): dielectric strength and space charge characteristics. IEEE International Conference on Solid Dielectrics. Bologna, pp363-365, 2013. 